\title{
The Impact of Extremely Large Telescopes on the Study of the Most Luminous Stellar Objects
}

\author{
Sandro D'Odorico \\ European Southern Observatory \\ K. Schwarzschild Str. 2, 85748 Garching bei Mnchen, Germany \\ email: sdodoric@eso.org
}

\begin{abstract}
The potential advantages of the new generation of Extremely Large Telescopes are briefly summarized. When used in combination with advanced adaptive optics modules which can substantially remove the effect of atmospheric turbulence at infrared wavelengths, these telescopes will provide unique capabilities both in terms of photon collecting power $(\rightarrow 2-4$ magnitude advantage) and angular resolution (4-5 times higher than with current 8-10m telescopes). The instruments under study for the TMT and E-ELT projects are presented and compared. I discuss the impact of the ELTs on three major science topics: stellar populations in galaxies to the Virgo distance, chemical abundances of the brighter stars in nearby galaxies and high redshift SN and GRBs.
\end{abstract}

Keywords. instrumentation: adaptive optics - galaxies: stellar content - supernovae: general gamma rays: bursts - telescopes

\section{ELTs: context and potential advantages}

In the last 25 years as many as 15 new $8-10 \mathrm{~m}$ telescopes have entered or are about to enter into operation. This gigantic increase in photon collecting power has had already a major impact on many field of astrophysics. It has pushed the brightness limit of the objects which can be studied 2-3 magnitudes fainter and has led to much higher S/N data in other types of observations. Beside the increase in the area of the telescopes, two other parallel developments had an equally positive influence. First, array detectors for both UV-Visual and NIR wavelengths have become regularly available in size up to $2 \mathrm{~K} \times$ $2 \mathrm{~K}$ pixels (for CCD up to $4 \mathrm{~K} \times 2 \mathrm{~K}$ ) and with $\mathrm{QE}$ above $60 \%$ over the whole range of sensitivity. Secondly, through the introduction of active optics pioneered by ESO at the NTT in the late 80 's, the optical image quality of the telescope has matched the best seeing achievable at the high quality sites (that is down to $0.3^{\prime \prime}$ ). Finally, in the last decade an additional observing capability has been demonstrated at the telescope and is now operating on a regular basis: the acquisition of images corrected for atmospheric turbulence by use of adaptive mirrors (e.g. NACO, SINFONI at the VLT, OSIRIS at Keck). These instruments are competitive in the NIR with the observing modes offered by the Hubble Space Telescope (HST) which operates above the atmosphere. Extremely Large Telescopes (ELTs) with diameters of the primary mirror larger than $20 \mathrm{~m}$ have now become the subject of detailed feasibility studies. The new telescopes will offer an order of magnitude increase in collecting area and they are going to rely on the use of adaptive optics to perform at the diffraction limit $(\theta=1.22 \lambda / \mathrm{D})$ over a moderate field with high Strehl ratios (concentration of light in the diffraction peak with respect to the total light from the source) at infrared wavelengths. The large diameter of the primary 
mirror of the ELTs will boast the angular resolution capability with respect to the spacebased James Webb Space Telescope (JWST), which is expected to start to operate in 2015 , has the advantage of the absence of a blurring atmosphere but has a diameter of $6.5 \mathrm{~m}$ only. Many classes of scientific objectives which rely on observations at the highest angular resolution(e.g. planetary discs, regions of star formation, active galactic nuclei) will benefit from this unique advantage. Beside the gain of the unique angular resolution when operating close to the diffraction limit of the telescope, the advantages of the ELTs with respect to the 8-10 m class ground-based telescopes can be quantified as follows, depending on the nature of the targets and the type of observations:

For photon-noise dominated observations, the faintness limit (at a given integration time and for a chosen $\mathrm{S} / \mathrm{N}$ ) and the speed (defined as the reciprocal of the time needed to reach a given $\mathrm{S} / \mathrm{N}$ for a given magnitude) are proportional to $\mathrm{D}^{2}$. When comparing the European Extremely Large Telescope (now nominally $42 \mathrm{~m}$ ) with the VLT UT ( $8 \mathrm{~m}$ ) this translates into a gain of 3.5 magnitudes. This regime corresponds e.g. to high time resolution or high spectroscopy resolution observations of bright sources.

For detector noise dominated observations, the faintness limit is proportional to $\mathrm{D}^{2}$, the speed to $\mathrm{D}^{4}$. This is a regime where the advantage of the ELTs is more relevant but with the improvement in the r.o.n. of modern detectors, this case is confined to the observations at high resolution spectroscopy at low $\mathrm{S} / \mathrm{N}$.

For sky background limited observations, at natural seeing image quality, the faintness limit is proportional to $\mathrm{D}$, the speed to $\mathrm{D}^{2}$. This points out that for this type of observations (e.g. large field imaging at red or NIR wavelength) the gain from the VLT $8 \mathrm{~m}$ UT to a $42 \mathrm{~m}$ telescope is less than two magnitudes for a fixed exposure time. An imaging survey with an $8 \mathrm{~m}$ telescope of large field (1 square degree of larger) can be more efficient than one carried out at the ELT with a field of a few square minutes. For operation at the telescope with an adaptive optics module which delivers images at the diffraction limit with a significant fraction of the flux of point-like sources within the Airy diffraction disc (high Strehl ratio), then the advantage of the $42 \mathrm{~m}$ in sky backgroundlimited observations becomes more relevant. The faintness limit is again proportional to $\mathrm{D}^{2}$ and the speed is proportional to $\mathrm{D}^{4}$. This regime corresponds to the observation of faint stellar sources like stars in external galaxies, GRBs and supernovae. By combining the intervals of best natural seeing with the power of $\mathrm{AO}$, it becomes possible to get observations of stellar fields which in limiting magnitudes are as deep as expected from the JWST but with an higher angular resolution. The gain in the observations of sources like high $\mathrm{z}$ galaxies which are not stellar but can be made up by sub-seeing knots will be lower but still relevant. It is important to note however that the AO modules of first generation will deliver DL performance at IR wavelengths only and over a field of less than 1 arcmin.

\section{The European ELT}

ESO is carrying out a Phase B study of a $42 \mathrm{~m}$ segmented primary telescope with the goal to prepare a construction proposal by the beginning of 2010. The European ELT current baseline foresees a 5 mirror optical design which delivers a 10' field at the Nasmyth foci with diffraction-limited image quality and $9 \%$ vignetting (Fig. 1, Delabre 2008). The optical train includes one adaptive mirror, M4, and a fast tip-tilt correcting mirror, M5, which can provide a correction of the ground-layer atmospheric turbulence and of wind buffeting on the telescope at the observatory site. Instruments at the E-ELT will be used either directly at the telescope focus (providing just a ground-layer correction with M4GLAO mode) or in combination with additional adaptive mirrors in a post-focal module 


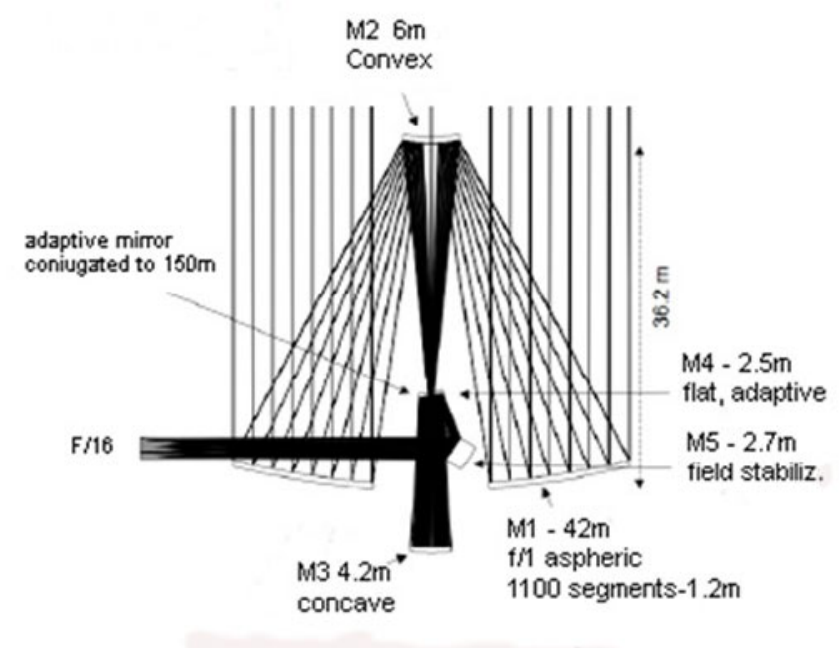

Figure 1. Basic Optical Parameters of the E-ELT baseline configuration

to obtain a diffraction limited performance with high light concentration over fields up to 1-2' at the Nasmyth foci. Fig. 2 and 3 show possible layout of instruments on the two Nasmyth platforms and on the additional coudè focus, which provides a protected location for instruments requiring a large volume and a controlled environment. This simulation shows that we could have up to 7 instruments permanently mounted at the telescope, offering a wide range of observing capabilities on line. This will permit the implementation of a flexible scheduling at the telescope to optimize the use of observing time and the scientific output. In the current Study Phase B of the project, ESO has launched 6 instruments and two post focal adaptive optics studies with institutes or consortia of institutes in the ESO member states. The choice of the instruments was based on the science case for the $42 \mathrm{~m}$ telescope as initially identified in the report by the E-ELT Science Working group (http://www.eso.org/sci/facilities/eelt/publ/ELT-SWGapr30-1.pdf) and on the basis of concept studies of instruments carried out in 2005-2006 at ESO and in other institutes under the European Community FP6 ELT Design Study program.

\section{A comparison of the first light TMT versus E-ELT Instrumentation}

The Thirty Meter Telescope (TMT) is a project of an optical-infrared telescope carried out in collaboration between CALTECH, the University of California and Association of Canadian Universities for Research in Astronomy (ACURA). Among the ELTs projects, the TMT is the more advanced in terms of elaboration of the science case and of the technical studies. A construction proposal and the supporting science case have been recently released (http://www.tmt.org/foundation-docs/index.html ). The TMT design foresees a 3 mirror Ritchey Chretien telescope, with two wide Nasmyth platforms which can host a suite of permanently mounted instruments. Unlike the E-ELT the TMT has no AO mirrors in its baseline optical train but includes a first light AO module (NFIRAOS) which will feed DL images to two of the first light instruments. Table 1 shows a 


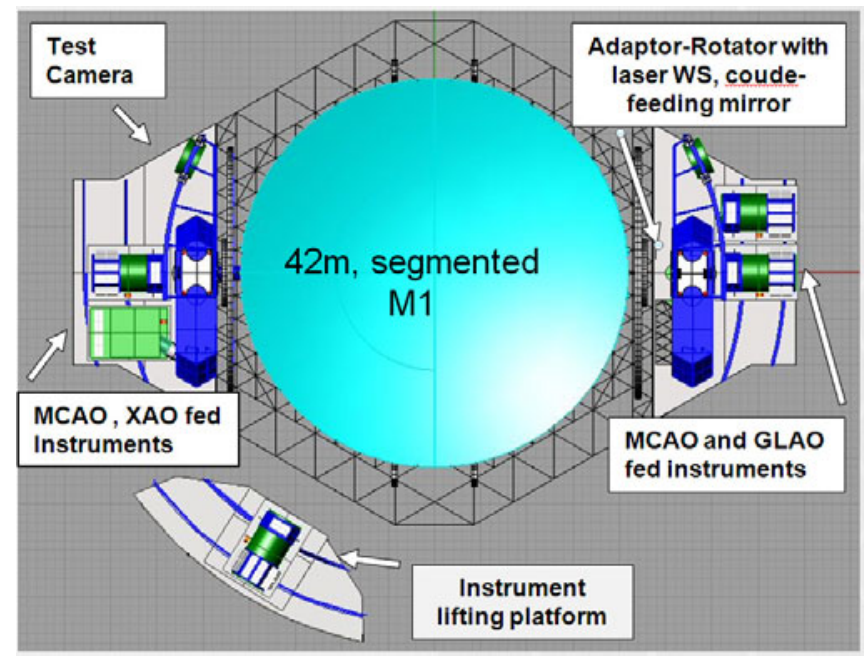

Figure 2. A top view of the two Nasmyth platforms of the European ELT showing a possible arrangement of 4 instrument and the adaptive optics modules.

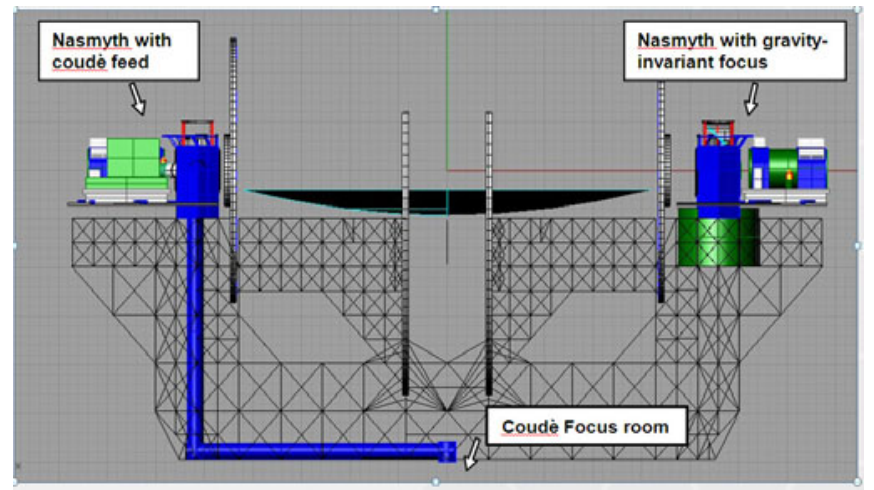

Figure 3. A side view of the European ELT showing the adaptors, AO modules and instruments mounted on the Nasmyth focus and the path to the coudè focus room. The gravity-invariant focus below the platform is foreseen for large field instrument which need to rotate with the sky but need to minimize flexures.

comparison of the initial requirement of the TMT and E-ELT instruments of first generation. In the case of the TMT, the project has identified two categories of instruments: first light instruments and instruments to be installed in the first decade of operation. For the E-ELT the 6 instruments now under detailed studies are candidates for first generation. Two additional instrument concepts will be selected in 2008 for advanced study and a selection will take place at the beginning of 2010 only, when the E-ELT construction proposal will be presented. There are many similarities and a few differences between the two suites of instruments. Both include a multi conjugate adaptive optics module to provide diffraction limited images in the NIR over a moderate field $\left(30^{\prime \prime}-2^{\prime}\right)$. This module can be coupled with an imager (IRIS at the TMT, MICADO at the E-ELT) and a spectrograph (again IRIS, HARMONI at the E-ELT) to take advantage of the unique size of the telescope from the early days of operation. Both telescopes foresee multi-object spectrographs working in the spectral range 800-2400 nm with AO, with the observations of galaxies at intermediate and high redshift as main driver (IRMS, EAGLE). Both 
Table 1. E-ELT and TMT Instrumentation

\begin{tabular}{|c|c|c|c|}
\hline & | E-ELT INSTRUMENT & & | TMT INSTRUMENT \\
\hline $\begin{array}{l}\text { EAGLE } \\
\left(1^{\text {st }} \text { gen }\right. \\
\text { candidate })\end{array}$ & $\begin{array}{l}\text { Wide-field, multi IFU NIR spectrograph } \\
+\mathrm{AO} \text { Wavelength range: } 0.8-2.5 \mathrm{~mm} \\
\text { FoV }>=5 \text { '; Multiplexing }>20 \\
\text { Spectral } \mathrm{R}=5000(\mathrm{R}>15000 \text { option }) \\
\text { IQ: }>30 \% \text { EE in } 100 \mathrm{mas}\end{array}$ & \begin{tabular}{|l|} 
IRMS \\
$($ early \\
light)
\end{tabular} & $\begin{array}{l}\text { NIR multi-slit spectrometer-imager } \\
\text { (clone of MOSFIRE @ Keck) } \\
\text { FoV }: 2.27 \text { arcmin Multiplexing: } 46 \\
\text { Spectral R=3270 }-4500 \\
\text { Spectral coverage: all of Y to K }\end{array}$ \\
\hline $\begin{array}{l}\text { MICADO } \\
\text { (1st gen } \\
\text { candidate) }\end{array}$ & $\begin{array}{l}\text { High angular resolution camera } \\
\text { Wavelength range: } 0.8-2.4 \mathrm{~mm} ; \\
\text { FoV:=>30" } \\
\text { operation with GLAO, LTAO, MCAO }\end{array}$ & \begin{tabular}{|l|} 
IRIS \\
$($ early \\
light)
\end{tabular} & $\begin{array}{l}\text { IFU NIR spectrometer and imager } \\
\text { Wavelength range: } 0.8-2.5 \mathrm{~mm} \text {; } \\
\text { FoV }<2^{\prime \prime} \text { for IFU }, 10 \times 10 \text { for Imaging; } \\
\text { Spectral } \mathrm{R}=4000(\mathrm{~J}, \mathrm{H}, \mathrm{K})\end{array}$ \\
\hline $\begin{array}{l}\text { HARMONI } \\
\text { (1st gen } \\
\text { candidate) }\end{array}$ & $\begin{array}{l}\text { Single field wide band spectrograph } \\
\text { Wavelength range: } 0.8-2.4 \mathrm{~mm} ; \\
\text { FoV: tbd } \\
\text { Spectral R: R } \sim 4000(\mathrm{R} \sim 20000) ; \\
\text { GLAO, MCAO modes }\end{array}$ & $\begin{array}{l}\text { WFOS } \\
\text { (early } \\
\text { light) }\end{array}$ & $\begin{array}{l}\text { Wide-field Optical MOS - seeing limited } \\
\text { Wavelength range: } 0.34-1.0 \mathrm{~mm} ; \\
\text { FoV }: 40.5 \text { arcmin } 2 ; \\
\text { Spectral } \mathrm{R}=500-5000\end{array}$ \\
\hline $\begin{array}{l}\text { CODEX } \\
\text { (1st gen } \\
\text { candidate) }\end{array}$ & $\begin{array}{l}\text { High-resolution visual spectrograph } \\
\text { Wavelength range: } 0.40-0.69 \mathrm{~mm} ; \\
\text { Spectral Res }>120000 ; \\
\text { Stability: }<5 \mathrm{~cm} / \mathrm{sec}\end{array}$ & $\begin{array}{l}\text { HROS } \\
\left(1^{\text {st }}\right. \\
\text { decade })\end{array}$ & $\begin{array}{l}\text { High-resolution visual spectrograph } \\
\text { Wav. range goal: } 0.31-1.0 \mathrm{~mm} \\
\text { Spectral resolution: } \mathrm{R}=50000 \text { (1" slit) }\end{array}$ \\
\hline $\begin{array}{l}\text { MIDIR } \\
(1 \text { st gen } \\
\text { candidate })\end{array}$ & $\begin{array}{l}\text { Mid-IR imager and spectrograph }+\mathrm{AO} \\
\text { Wavelength range: } 7-20 \mathrm{~mm} \\
\text { (goal 3.5-27 mm) } \\
\text { FoV: =>30" diameter } \\
\text { Spectral R: tbd }\end{array}$ & $\mid \begin{array}{l}\text { MIRES } \\
\left(1^{\text {st }}\right. \\
\text { decade })\end{array}$ & $\begin{array}{l}\text { Mid-IR imager and spectrograph }+\mathrm{AO} ; \\
\text { Wavelength range: } 8-18 \mathrm{~mm} ; \\
\text { FoV: } 10 " \\
\text { Spectral R: } 5000<\mathrm{R}<100000\end{array}$ \\
\hline $\begin{array}{l}\text { EPICS } \\
\text { (1st gen } \\
\text { candidate) }\end{array}$ & $\begin{array}{l}\text { Planet imager and spectrograph } \\
\text { Coupled to XAO, coronograph } \\
\text { Wavelength range: } 0.6-1.8 \mathrm{~mm} ; \\
\text { Spectral R }>50 \text { in Y to } \mathrm{H} \text { bands, } \\
\text { Polarimetry }\end{array}$ & $\begin{array}{l}\text { PFI } \\
\left(1^{\text {st }}\right. \\
\text { decade })\end{array}$ & $\begin{array}{l}\text { Planet imager and spectrograph } \\
\text { Coupled to XAO, coronograph } \\
\text { Wavelength range: } 1-2.5 \mathrm{~mm} \\
\text { Spectral } \mathrm{R}=50 \text { full FOV } \\
\mathrm{R}=500 \text { partial FOV }\end{array}$ \\
\hline
\end{tabular}

include an instrument dedicated to imaging/spectroscopy of planets close to bright stars (PFI, EPICS) and MID Infrared instruments (MIRES and MIDIR). High resolution visual spectrographs have been studied for both projects: HROS - a first decade TMT instrument- is a classical echelle aiming at a resolution of 50000 while CODEX for the E-ELT is optimized for high stability, high accuracy radial velocity measurements. TMT is the only project to include a blue-visual, wide field MOS spectrograph optimized for operation with natural seeing. Among the first decade TMT instruments, but not included in Table 1 are also an Infrared Multi Object Spectrometer (IRMOS, a scaled up version of IRMS) and a Near Infrared Echelle Spectrometer (NIRES).

\section{The impact of ELTs on the study of the most luminous stellar objects}

In the previous sections the potential advantages of the new generation of ELTs have been presented and the current concepts of the first generation instruments for the two more advanced projects, the TMT and the European ELT have been compared. Most of the instrument studies have not yet been finalized. Those for the E-ELT are in their initial phase of the detailed design and their impact on specific scientific areas has not yet been accurately quantified. We consider below three topics related to the subject of this Symposium where the predicted advantages of observations with the ELTs are outstanding:

Color-Magnitude diagrams of stars in external galaxies. The angular resolving power of an ELT used in combination with AO will be uniquely suited to do accurate photometry 


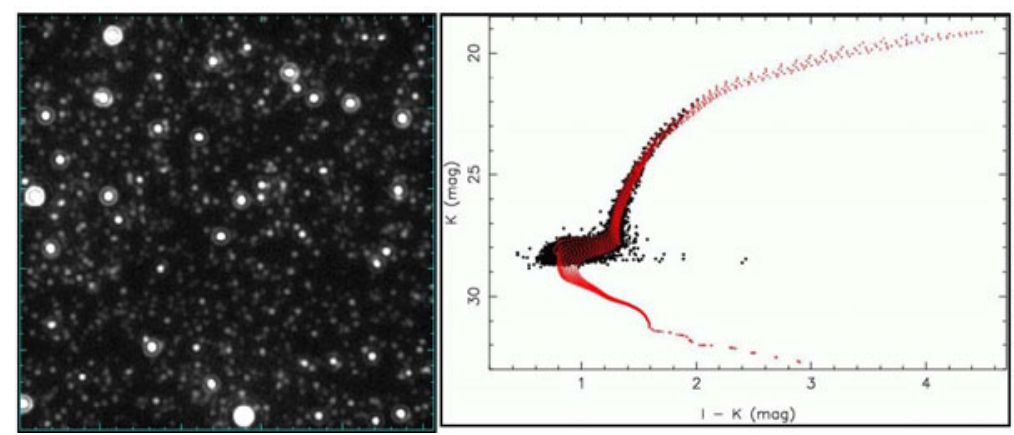

Figure 4. Left: Simulated K image of a stellar population of an elliptical galaxy at $\mathrm{DM}=26$, with a V surface brightness of $22 \mathrm{mag} / \operatorname{arcsec}^{2}$. The image area is $\sim 10 \operatorname{arcsec}^{2}$, observed with the $42 \mathrm{~m}$ E-ELT for 100hrs. Right: input stellar population (light circles) and extracted CMD (darker circles). From Liske (2007).

of stellar populations in external galaxies. It will provide for the first time both the depth and the angular resolution to build up color magnitude diagrams well beyond the Local Group. These could be used to reconstruct the history of star formation in galaxies of different morphological type than of our own galaxy. Measurements of the bright part of the CMD of elliptical galaxies as far as Virgo ( $\mathrm{DM}=31$ ) and the HB to Centaurus $\mathrm{A}(\mathrm{DM}=26)$ are among the science drivers of instruments like IRIS at the TMT and MICADO at the E-ELT. Liske (2007) at ESO has carried out a first set of simulations of crowded stellar fields in external galaxies using the predicted PSF of the telescope with an adaptive optics module and an assumed stellar populations. Different photometry packages have been used on the simulated images to extract the photometric measurements from which the CMD has been reconstructed (Fig. 4). This exercise is essential to investigate the ultimate capabilities of the telescope and to optimize both the instrument parameters and the data reduction software.

Chemical abundances in stars of different stellar populations. High and intermediate resolution spectrographs at 8-10m telescopes (e.g. UVES and FLAMES+GIRAFFE at the ESO VLT, HIRES at Keck) have been used very effectively in the last decade to extend our knowledge of the abundances in stellar populations in our Galaxy and in other members of the Local Group (Tolstoy 2007 and references therein). The observations do hint to a complex star formation history even for galaxies which have a relatively simple morphological structure like the Local Group dwarfs. This type of observations is essentially photon-limited and has been confined to the most luminous stars in each system. They will benefit from the improvement of more than one order of magnitude in collecting area provided by the ELTs and in any improvement in angular resolution to resolve the brightest stars in the crowded regions as it could be obtained with a small field AO system. The expected gain is of 3-4 magnitudes, making possible measurements for RGB in Local Group galaxies. Of the instruments under study for the TMT and the E-ELT, HROS appears as the best option for the high resolution work. The NIR multi-object spectrographs like IRMS and EAGLE could be used very effectively in the statistical study of the Ca II triplet as metallicity indicator (Fig. 5) in a large sample of extragalactic stars like it has been done at brighter magnitudes with $8-10 \mathrm{~m}$ telescopes (Battaglia et al. 2008). Here it is essential that the spectral range of the ELT NIR spectrographs will include the I band. The gain with the ELT at these wavelengths can be as high as 4-5 magnitudes because the observations will benefit from the light concentration provided by a wide field GLAO or MOAO system associated to the telescope. 


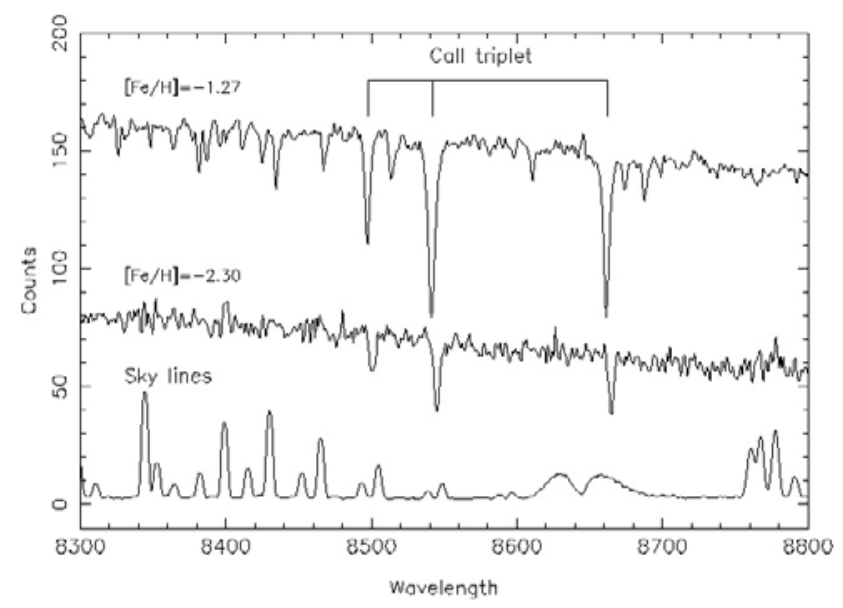

Figure 5. Observations of the Ca II triplet at intermediate resolution have been shown to be effective in measuring the metallicity of stars in different stellar populations (Battaglia et al. 2008).

The WFOS instrument at the TMT is potentially a powerful instrument to extend the work carried out on supergiants stars in the Local Group spirals and dwarf irregulars (Urbaneja et al. 2008, Bresolin et al. 2008, and references therein) to fainter magnitudes and to galaxies beyond the Local Group, up to $10 \mathrm{Mpc}$. It has been demonstrated that by comparing intermediate resolution spectroscopy with model simulations, it is possible to derive both the physical parameters and the metallicity of the brightest stars. These can be complemented by the gaseous phase abundances from the emission lines in the H II regions. A multi object spectroscopy capability is a strong requirement for this program. The observations would also greatly benefit from any improvements in the angular resolution but a modest gain can be expected at the visual wavelengths of this instrument from AO. Observations with WFOS will have to rely on the best natural seeing of the site.

Near-Infrared Spectroscopy of faint stellar sources. Both the TMT and the E-ELT instrument suites feature faint object spectrographs which will be operated in combination with AO modules pushing the limit of spectroscopy in the NIR to magnitude as faint as 28. Stellar sources like $\mathrm{SN}$ up to $\mathrm{z}=2$ and GRBs at redshifts up to $\mathrm{z}=10$ are prime targets for single field spectrographs like IRIS (TMT) and HARMONI (E-ELT). These observations are expected to have a major impact on a large spectrum of fields of astrophysics, from the last phases of stellar evolution to star formation, from cosmology to the properties of the IGM.

\section{References}

Battaglia, G., Irwin, M., Tolstoy, E., et al. 2008, MNRAS, 383, 183

Bresolin, F., Urbaneja, M. A., Gieren, W., et al. 2008, ApJ, 691, 2028

Delabre, B. 2008, $A \mathscr{\varepsilon} A$, in press

Liske J. 2007, http://www.eso.org/sci/facilities/eelt/science/drm/

Tolstoy E. 2007, in: A. Vazdekis \& R. F. Peletier (eds.), Stellar Populations as Building Blocks of Galaxies (Cambridge: CUP), Proc. IAU Symp 241, 279

Urbaneja, M. A., Kudritzki, R. P., \& Bresolin F. 2008, in: S. Koribalski \& H. Jerjen (eds.), Galaxies in the Local Volume, (Berlin: Springer), Astrophys. Space Sci., in press 


\section{Discussion}

WALBORN: Has the exact size of the E-ELT been selected yet?

D'Odorico: In the present Phase B study to be completed by the end of 2009 the baseline concept foresees a $42 \mathrm{~m}$ segmented primary mirror.

MARTAYAN: I did not see in your talk 2 instruments for the E-ELT initially foreseen for the first generation and described in earlier ESO documents: a multi-object spectrograph like a super VLT FLAMES and a spectro-polarimeter . These instruments, according to me, seem to be extremely important to obtain information and statistics on stars of the galaxies beyond the local group. Are they definitely removed from the 1st generation instruments if the E-ELT or could we influence yet ESO?

D'Odorico: The two instruments you quote are indeed not included in the six currently under study. However they could be proposed in response to the Call for two additional instrument concept studies that ESO will release in February 2008. A decision on the first generation E-ELT instrumentation will not be taken before the beginning of 2010 . 